\title{
An Historical Analysis of the SEAndroid Policy Evolution
}

\author{
Bumjin Im Ang Chen Dan Wallach \\ Rice University, Houston, Texas, USA \\ December 4, 2018 \\ \{bi1, angchen, dwallach\} @ rice.edu
}

\begin{abstract}
Android adopted SELinux's mandatory access control (MAC) mechanisms in 2013. Since then, billions of Android devices have benefited from mandatory access control security policies. These policies are expressed in a variety of rules, maintained by Google and extended by Android OEMs. Over the years, the rules have grown to be quite complex, making it challenging to properly understand or configure these policies.

In this paper, we perform a measurement study on the SEAndroid repository to understand the evolution of these policies. We propose a new metric to measure the complexity of the policy by expanding policy rules, with their abstraction features such as macros and groups, into primitive "boxes", which we then use to show that the complexity of the SEAndroid policies has been growing exponentially over time. By analyzing the Git commits, snapshot by snapshot, we are also able to analyze the "age" of policy rules, the trend of changes, and the contributor composition. We also look at hallmark events in Android's history, such as the "Stagefright" vulnerability in Android's media facilities, pointing out how these events led to changes in the MAC policies. The growing complexity of Android's mandatory policies suggests that we will eventually hit the limits of our ability to understand these policies, requiring new tools and techniques.
\end{abstract}

\section{Introduction}

Smartphones are a primary target of malicious attacks [21]. The Android system-as it holds the largest share of the mobile OS market-has unfortunately become a prominent attack target as well [19]. Over the years, many attacks have been reported, such as Stagefright [52], Blueborne [3], and Toaster [34], each of which has led to significant security concern. In order to harden Android against such threats, researchers and developers have adapted a wide variety of security mechanisms to the Android envi- ronment, including process separation, finer grained access control, and secure booting / remote attestation. This study focuses on one such mechanism: SEAndroid [43].

As an extension of SELinux [29], SEAndroid was originally introduced by the NSA in 2013. It performs mandatory access control (MAC) to enforce security policies, regulating whether a particular subject (e.g., a process) can perform a certain action (e.g., read/write) on an object (e.g., a file/socket). It achieves this by referring to a pre-installed security policy with a set of access control rules, which are compiled into a database and loaded by the kernel at boot time.

As with SELinux, configuring a SEAndroid policy is not an easy task [16, 41]. It is often far from obvious to reason about whether a particular set of rules achieve a desired policy, or even to understand what policy certain rules try to implement. There are several reasons for this. First, the policy language allows many abstraction features, such as groups, attributions, and (nested) macros, which make it challenging to infer the scope of individual rules. Second, the policy rules have little accompanying documentation. Moreover, they evolve significantly every year.

Fortunately, Google's default SEAndroid policy is maintained in a Git repository [1] as part of the Android Open Source Project (AOSP) [12]. The Git history provides us with a detailed chronicle of all changes to the policy rules, as well as the commit messages associated with each change, totaling more than 13,000 commits over SEAndroid's history. These commits serve as a rich source of information for us to perform an historical analysis of the policy's evolution, snapshot by snapshot.

Performing this analysis involves at least two challenges. First, we need a good metric to quantify the complexity of a particular policy snapshot. Simply counting the number of rules in a snapshot is not enough, because the SEAndroid policy language supports a variety of abstractions and grouping concepts, making a rule count less useful. We address this challenge by designing a new metric, boxes. This metric views all possible access control policy rules as forming a four-dimension space, with the axes being sub- 
ject, object, class, and permission. Each point in this space is called a box, representing a smallest "unit" in the rule space. By analyzing how many rules touch the same box, and how many boxes are impacted by any given rule, we can then quantify the policy's complexity. Consider that complex rules may touch many boxes and many of those boxes may be impacted simultaneously by multiple rules. This means that a change to any one rule may or may not result in a change to the resulting security policy! If an engineer truly desires to change a given box, it becomes necessary to go hunting for every possible rule that might overlap with it.

Our second challenge is that there are thousands of Git commits that impact the SEAndroid policies. We use a combination of approaches to address this. We have designed and implemented an automated system that collects and analyzes each Git commit, identifying "jump points" in complexity both in terms of the number of rules and the number of boxes. For significant jump points, we also manually inspect the commit messages associated with these commits, and perform a differential analysis on the rules before and after each such commit to understand the rationale of the changes. Furthermore, we use the timestamp information to "match" the commits with historical events of Android security, and analyze how these events are reflected in the policy's evolution.

Using the above metrics and methodology, we have performed an historical analysis on the SEAndroid policy's evolution. We focus on the development of security metrics that we can derive from these policies, such as the number of boxes and rules, the evolving list of types and macros, the different authors contributing to the policy over time, how SEAndroid policy stabilized over time, as well as the hallmark events in Android history. Based on these measurement results, we also provide insights into how SEAndroid might evolve to become simpler and more useful.

The structure of the paper is as follows. After describing more background material in Section 2, we introduce our measurement methodology in Section 3 , present measurement results in Sections 4 and 5 . Then, we discuss several related topics in Section 6 , present related work in Section 7, and conclude in Section 8

\section{Background}

In this section, we present more background material on Android security architecture and, in particular, SEAndroid.

\subsection{Android security architecture}

Figure 1 shows the multiple layers of security mechanisms Android uses to protect system resources and user data [7].
Install-time permissions. Every Android application includes a "manifest" file specifying the app's desired permissions. Prior to Android 6.0, the user was queried at install-time whether the desired permissions were acceptable (i.e., all-or-nothing). After this, the new app is assigned a distinct Unix "user" ID, allowing traditional Unix-style file permissions to separate the storage for each app. However, Android includes a variety of system services, speaking over Binder (an interprocess communication channel mediated by the OS kernel). Each Binder service is responsible for determining if its caller is permitted to use it, querying a central database constructed from those installtime permissions. This resulted in permission checks occurring all over the Android software base, in both Java and native code. This, in turn, made it a challenging research project just to produce a mapping from every Android API call to its corresponding set of required permissions [8].

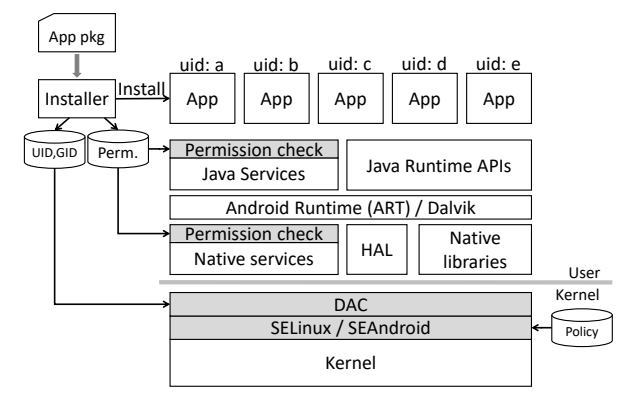

\section{Figure 1. The Android security architecture.}

Time-of-use permissions. As of Android 6.0, Android apps still declare their permission requirements in the manifest, but those permissions are not granted until the app explicitly requests them from the user, preferably at time-ofuse. Users are free to deny permissions and even to revoke permissions later on from the system settings. The underlying enforcement of these permissions is largely the same as before, with individual Android services querying whether their caller has a necessary permission.

Classic Unix discretionary (DAC) permissions. Of course, Android is also just another flavor of Unix. If an application directly accesses a Unix resource such as a file or device, traditional Unix user and group IDs manage the security. A Unix group ID is preassigned to each permission, and the Android application launcher assigns all the necessary group IDs to the application process when it is launched. Since a Linux process can possesses multiple group IDs, the access will be granted or denied appropriately.

SEAndroid mandatory (MAC) permissions. After the user ID and group ID checks are performed, SEAndroid [43], which is an extension of SELinux [29] for Android, can additionally check all system calls against its own 


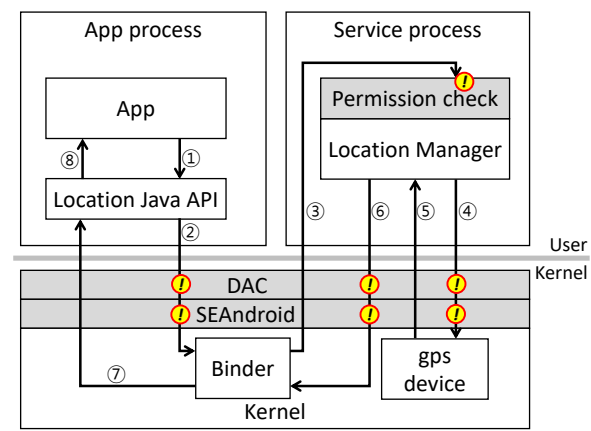

\section{Figure 2. Control flow with the Android loca- tion API. Seven permission checks are high- lighted with yellow circles.}

policy. This policy is loaded at boot time and enforced inside the OS kernel. SEAndroid policies, by their static nature, cannot be changed at runtime to reflect new applications and user-expressed permissions. They can, however, be used to isolate system services, file directories, and even Binder resources.

\subsection{Example: Location services}

To show how these different security systems interact, Figure 2 diagrams the control flow involved when an app makes a call to the Location Services, which manages the GPS device and other location-related (and thus, privacysensitive) features.

If an application calls a location API (1), the corresponding library establishes a Binder channel between the application and the location manager (2). Since Binder is an IPC mechanism provided by the kernel, the client requires a corresponding discretionary access control (DAC) permission which is always allowed. After that, a SELinux hook is called to check the SEAndroid permission, which is also configured to always permit this call. Binder transfers the request to the location manager (3), which then calls a checkPermission() system API (shown as "permission check") which checks if the user granted the relevant location permissions to the calling app. If granted, the location manager interacts directly with the GPS device (4). However, this access also requires DAC and SEAndroid enforcement, which are configured so no other subject in the Android universe is permitted to interact directly with the device. The API response unwinds the call path 5 - 8 . Each step includes another opportunity for DAC and SEAndroid permissions to be checked, but these are all permitted by default.

Even in this simple example, there are seven different access control enforcement opportunities on the path from the app to the GPS device driver. In this case, DAC and
SEAndroid only serve to ensure that the GPS device is only reachable from the location manager service, while the permission checking for the app is handled internally by the location manager. As this example illustrates, Android security enforcement is complex to analyze.

Here, the value of SEAndroid is to protect system resources more precisely from low-level attacks. But as we see in Figures 1 and 2, the SEAndroid policy does not have any relationship to the Android security model as Android users and app programmers understand it. In addition, there is no formal documentation specifying any of the SEAndroid policies, much less how they interact and how the user-visible permissions or Unix discretionary permissions are meant to interact with each other and with SEAndroid.

\subsection{SEAndroid policy rules}

The SEAndroid policy engine was present on Android devices since the early 2010s, but it was configured in an inactive "permissive mode". The policy was initially configured by NSA and committed to the AOSP repository [1]. Any Android device OEM can add their own policy along with the original policy, e.g., using tools such as setools [45] and audit2allow [35]. This is typically done by examining denial logs and adding additional rules, which can then be submitted as patches for inclusion in future ASOP releases.

To enforce the policy, the rules are then parsed and compiled by a compiler called checkpolicy and loaded in the kernel at boot time. Needless to say, configuring a SEAndroid policy is critical, but at the same time, not an easy task [42,41]. A misconfigured policy will lead to misbehaviors or even security vulnerabilities. In order to understand the complexity of the policy, we need to first understand what the rules look like.

Allow rules. The majority of the policy consists of a sequence of "allow rules", such as allow appdomain zygote_tmpfs:file read, which states that any subject of the type appdomain (i.e., all applications) should be granted access to any file object of the type zygote_tmpfs (i.e., files that the zygote process created in the tmp file system). Besides file, typical classes also include directory, socket, process, and so on, each indicating a particular category of resources. This rule additionally specifies a read permission, meaning that accesses can only happen via the read system call. As we can see, such rules allow for very fine-grained policy, but writing a rule for each subject and object/class pair for every system call would not scale.

Abstraction features. To avoid the policy size from growing out of control, the SEAndroid/SELinux policy language supports a number of abstraction features, such as wildcards, groups, negation, and com- 
plements. A wildcard $(*)$ can be used in any object, class, or permission fields to represent "any possible entity". For instance, a rule allow appdomain zygote_tmpfs:file * would allow any system call, not just read. One could additionally group several entities together, such as allow appdomain zygote_tmpfs: file dir *. The complement feature is indicated using a tilde $(\sim)$, such as allow init fs_type:filesystem relabelto, meaning that any system call but a relabel to is permitted.

Neverallow rules. "Neverallow" rules works as assertions for debugging. If such a rule overlaps with an allow rule, the policy compilation would report an error. These rules have a similar format with allow rules, such as neverallow domain init:binder call.

\subsection{The complexity of SEAndroid policy}

Over the years, the SEAndroid policy has grown to be very complex. For instance, the snapshot as of July 2017 specifies 91 classes and 1,603 permissions. In addition to this, three more factors exacerbate the complexity.

First, the use of abstraction features only hide the complexity but does not eliminate it. For instance, allow untrusted_app self:file * may be easier to read, but the wildcard also provides an opportunity to sweep important issues under the table. Similar to abstraction features, SELinux supports DTE [4], which allows a domain to be associated with multiple types. This significantly decreases the number of rules, but again, hides complexity and potential vulnerability.

Second, the existence of neverallow rules itself hints at the complexity of the policy. In fact, SEAndroid is by nature a mandatory access control mechanism, which means that the default action for anything is already "disallow", unless explicitly granted access by an allow rule. Providing such assertion tool for policy configuration of a mandatory access control mechanism implies that there is concern for debuggability and for accidentally allowing too many actions.

Third, the policy engineering practice is at times idiosyncratic. One common practice of developers, for instance, is to extract denial messages from the kernel log using tools like audit2allow and simply create a new policy to allow them. Security concerns aside, this tends to create unoptimized, messy policy rules.

The combination of the above factors mean that it becomes difficult for an analyst to read the rules and understand exactly what a given local change might entail in the final calculus of what is allowed and what is denied. Such complexity, in fact, is one of the motivating factors for us to perform this analysis.

\section{Methodologies}

To the best of our knowledge, we are the first to perform an historical analysis of the SEAndroid policy. This is in contrast to previous work, such as Wang et al. [50] that use machine learning to improve the policy, and Zanin et al. [51] that use formal methods to verify policy correctness. Our goal is to understand not only the most recent snapshot, but also how the policy has evolved over time. Since its introduction, more than 16,000 commits have accumulated in the repository, with detailed timestamps, author information, and commit messages, providing a valuable source of information for understanding SEAndroid.

\subsection{The "box" metric}

One might wonder whether the complexity of a policy snapshot could simply be measured by counting the number of rules. However, this is not enough due to the abstraction features, such as macros, groups, and wildcards, that are heavily used throughout the policy base. To address this, we have designed a new metric, the "box", which is similar in spirit to Lampson's term "attribute" in his original work on access control matrices [22]. At a high level, a box is a quadruple with one subject type, one object type, one class, and one permission. This is the atomic unit that we use to quantify the complexity. With this, we can look at two interesting metrics: how many rules target each box, as well as how many boxes are targeted by each rule.

From rules to boxes. In order to obtain the boxes, we decompose each rule in the policy base in the following way. First, we expand all the macros used in the policy rules, and obtain all the classes and permissions from the access_vectors file. We then scan all the policy files and obtain all attributes and types. After this, we perform a second pass over all the policy files and decompose each allow and neverallow rule to their respective boxes, using the subject, object, class, and permission fields in each rule. A single allow rule may be decomposed into many allow boxes; neverallow rules are decomposed as "negative" boxes which are then subtracted from the allow boxes. The final outcome is a list of allow boxes equivalent to the original policy.

\subsection{Git repository analysis}

We repeat this analysis using boxes for each snapshot contained in the Git history. A Git history is a directed acyclic graph (DAG), where the commits are vertices and parent-child relations are edges. Because Git supports multiple branches which might then merge, not every pair of Git commits is necessarily going to have an ancestor-descent 
relationship. By analyzing the graph along with the contents of each vertex, we would be able to understand how the policy evolved over time. In addition to analyzing the source code "diff" in each commit, a commit also has various types of metadata, such as the committer's timestamp, email address, and comments. Such information allows us to gain further understanding of the rationale behind each policy change. Last but not least, based on the timestamps of the Git commits, we can also associate these changes to important historical events, such as security breaches.

One complication that arises due to the nature of Git is the large number of branches, as it is a distributed source code management system with many contributors. A contributor can create new branches any time without communicating with the master branch, and merge these branches back. Consequently, if we looked only at the timestamp of each commit and ordered every commit by these timestamps, we would be looking at multiple interleaved histories. Git also supports a "rebase" feature that allows separate branches to be rearranged into single linear timeline, albeit with the original timestamps, although multiple changes can also be "squashed" post-facto into a single commit event. Suffice to say that our view of the Git repository, as it's delivered to us, certainly represents the evolution of the SEAndroid policy over time, but it's possible that we're not seeing important parts of the history.

To simplify our analysis, we decided to perform our measurement study on the master branch only. Although looking at other branches may give additional information, we believe that an analysis of the master branch is a useful starting point, because the master branch reflects the history of Android as it was shipped from Google through AOSP to the Android OEMs.

\subsection{Our measurement tool}

We build our measurement tool in Python using 2,000 lines of code, with three components. Our crawler uses standard Git commands to check out the repository snapshots, our parser generates the box metrics from rules, and our serializer uses Python's msgpack library to store the policy to the disk.

On our experiment platform, an Intel Core i7 computer with 4 cores and 32 GB RAM and a 4 TB hard disk, our tool takes several seconds per policy snapshot, parsing all rules and generating boxes from the rules. For all commits from January 2012 to August 2018, our tool generates 3 TB of raw data, with hundreds of thousands of boxes per commit. Constructing the full database takes more than 90 hours of processing.

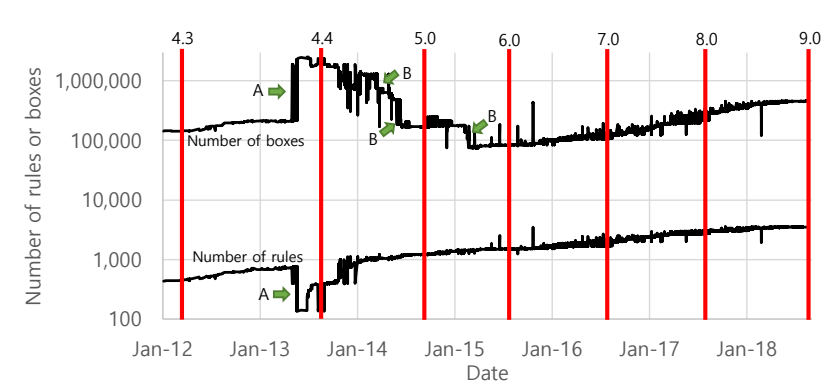

Figure 3. The number of rules vs. the number of boxes.

\section{Measurement Results}

In this section and the next, we present the measurement results obtained from 16,100 commits to the SEAndroid policy repository between January 2012 and August 2018. We focus on the results obtained using the new box metric in this section, and provide a broader, historical analysis in the next section.

\subsection{Boxes vs. rules}

Figure 3 shows two time series of the number of rules versus the number of boxes in each commit. We can see that, except for the period of time between mid-2013 and mid-2015, the curves are increasing roughly exponentially (note that the Y-axis is in logarithmic scale).

Zooming in on the timeline between mid-2013 and mid2015, we can see many fluctuations in both curves. We have in particular identified two events that we marked as " $A$ " and "B". In the "A" commit, authored by a Google engineer, the number of rules suddenly dropped from 1,000 to 200; but the number of boxes jumped from 214,000 to $2,315,000$, an increase of more than $10 \times$. We have manually analyzed this commit, as well as the differences from the previous commit, and found that this new commit associated all types to a single domain-unconfineddomain. This new domain allowed virtually any access to any object, and removed all other individual access rules with the same subjects and objects, which represents a clear break from past practice. Interestingly, we found that this "unconfined domain" was present even in the original version authored by NSA; but at that time, it was intended for some special entities that should bypass all security enforcement, and nothing was associated with it.

So what was Google trying to accomplish with this one commit? Here is the original commit message: 


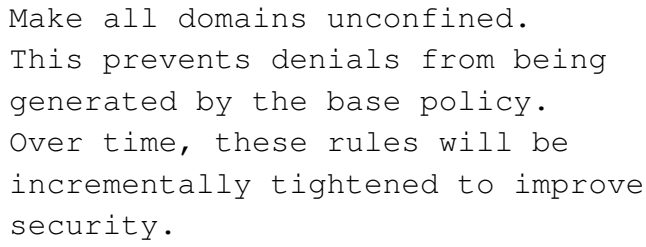

We are not able to find any external documentation for this drastic decision, but we could draw inferences about what problems they may have been facing. In 2012, Android passed iOS in market share [30], having roughly double the market share of iOS in 2013. Such drastic growth and intense competition may have created pressures to ship code on time, with security necessarily a lower priority. As the commit text suggests, Google had long-term plans for improving its use of SEAndroid, but for now wasn't planning on using it for anything beyond the benefit of having it integrated at all, allowing OEMs to begin experimenting with SEAndroid security policies.

Indeed, Google did fix it later. The "B" arrows indicate changes to the SEAndroid policy that drastically shrank the size of the "unconfined domain", and the general downward slope of the number of boxes during this time period shows a diligent effort over 1.5 years to ultimately eliminate the unconfined domain from the SEAndroid policy. By the Android 6.0 release, the unconfined domain was no longer in use.

We note the disconnect between the number of rules and the number of boxes. Each of the "B" arrows shows a significant reduction in the number of boxes, yet there is no corresponding change in the number of rules. This suggests an engineering process of methodically adding focused rules to cover the needs of various applications that were previously satisfied by the unconfined domain. After policy testing, large chunks of the unconfined domain could be unnecessary and therefore removed. We see this in several large downward steps in the number of boxes, as well as in the broader downward slope during this time period.

As of 2018, Android is even bigger than before, there are more participants than before, and the policy is no more changing rapidly, so we do not expect that a similar event is happening, but this is a great example for us to suggest we need proper metrics on the policy configuration as well as the documentation.

Takeaway \#1: Such results illustrate the importance of considering rules and boxes as distinct metrics of policy complexity. Even though in more recent years we see the rules and boxes growing side by side, there is demonstrably no necessity that there be a linear relationship between rules and boxes.

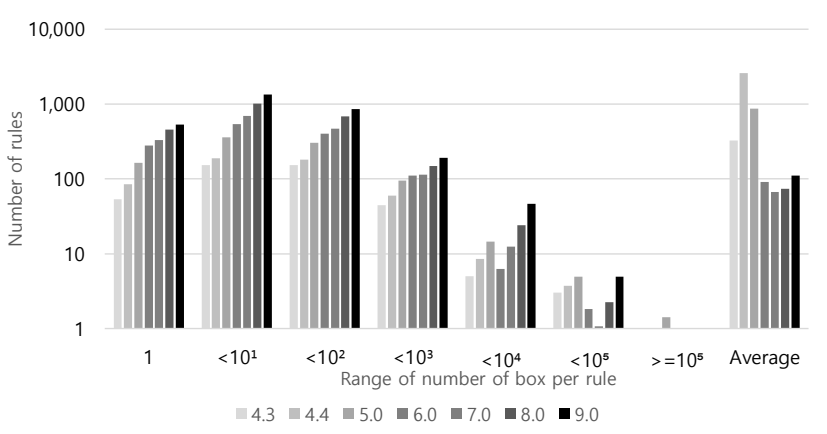

Figure 4. Average number of boxes per rule.

\subsection{Number of boxes in a rule}

As we noted earlier, the number of boxes or rules, by themselves, do not necessarily tell a complete story about the complexity of a given security policy. We next look at the number of boxes per rule, which measures the complexity due to the use of macros and other grouping features of SELinux security policies.

Figure 4 shows these ratios as of each major Android release from Android 4.3 through 9.0. We then group the results into frequency buckets on a logarithmic scale, so " $<$ $10^{1}$ " counts the number of rules that touch $2-9$ boxes, and " $<10^{2}$ " counts the number of rules that touch $10-99$ boxes. From this, we can see that we have similar numbers of rules that touch a single box as we have rules that touch $10-99$ boxes, with some falloff once we consider rules that impact 100 or more boxes.

For each frequency bin, we see an upward slope from Android 4.3 through to 8.0. Keep in mind that the $y$-axis is log-scaled, so these represent an exponentially growing number of rules in each bin, which is consistent with our earlier measurements in Figure 3.

There is an interesting data point in Figure 4. In Android 9.0, the bin $10000-99999$ has grown from 1 to 5 . The four new rules are:

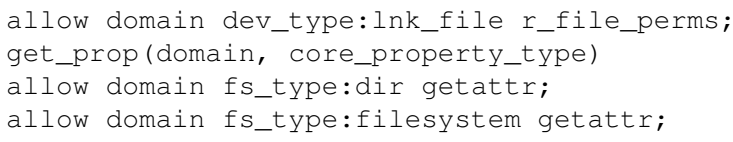

These four rules allow the attribute domain, which includes virtually every subject, to read all "link files" (i.e., symbolic links), to invoke the stat () system call on virtually every file and directories in the system, and to access all the "core properties" (i.e., configuration values similar to the registry in Microsoft Windows). In addition, even though there are only five rules in this bin, they impact more than 110,000 boxes. Therefore, if there is ever a need to deny the stat () call for a few specific processes, one would need to carve out additional rules from these generic 


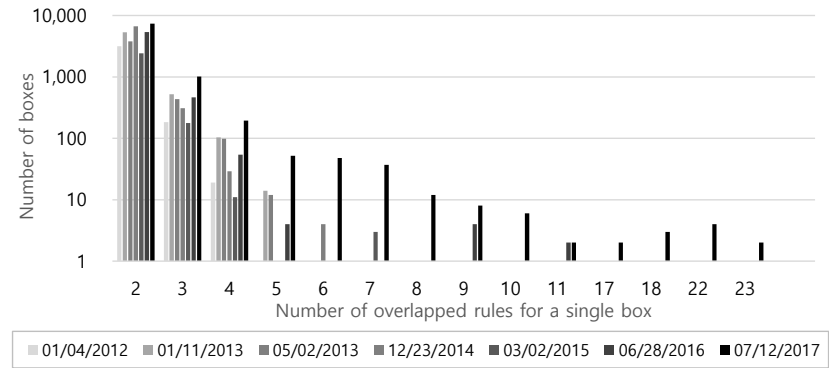

Figure 5. Number of rules mapped to a single box.

rules, e.g., by creating certain negative rules or splitting these generic rules into smaller, more specific ones.

Lastly, we present the "average" number of boxes per rule across each Android security policy snapshot. We see a spike in Android 4.4, which is likely caused by the massive "unconfined" domain issue. It then drops for 5.0, and becomes relatively stable from 6.0 through 9.0. This suggests that Android team has achieved a stable engineering discipline, in the sense that while the absolute number of boxes is growing exponentially, the effort per box to construct suitable policies is roughly constant across these three releases.

Takeaway \#2: Some small number of rules could touch a large number of boxes due to the abstraction features. Overall, the number of boxes touched by a rule increased over time, but this ratio seems to be stabilizing, despite the continued exponential growth in the absolute number of rules and boxes.

\subsection{Number of rules per box}

As before, we define a box to be an atomic access control unit. Our next concern is that multiple rules might speak about the same box. Why is it bad to have multiple rules influencing the same box? Consider the case of a security engineer, auditing the security policy, who determines that one of the rules is over-broad and thus requires some effort to fix the problem. If our engineer didn't realize that multiple rules allowed the same box, then this engineering effort might not have its desired impact. Where a high ratio of "boxes per rule" indicates the use of macros and grouping features, a high ratio of "rules per box" instead indicates a degree of imprecision in the design of the rules.

Figure 5 shows the rules per box for seven different commits at dates selected across the range of Android. Since it is natural that one box should be derived from the only one rule, we do not collect these cases. As we see from the graph, there are a large number of boxes derived from 2 or 3 rules for all the sampled commits. More importantly, there

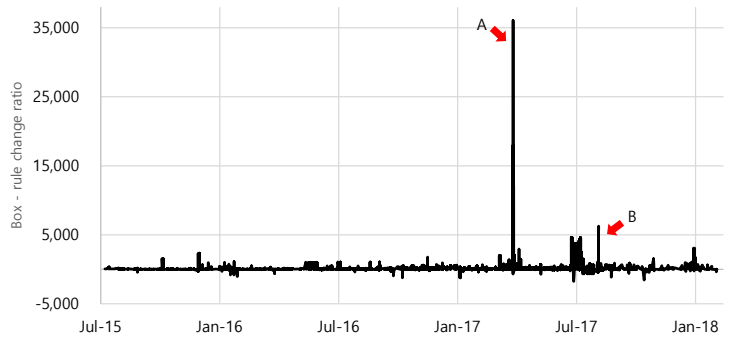

Figure 6. Ratio between change of rules and boxes.

is a trend that the tail (i.e., boxes with ten or more rules that specify them) is growing over time. The most popular box, generated by a remarkable set of 23 rules is:

system_server system_file:dir search;

Some example rules that generate this box include:

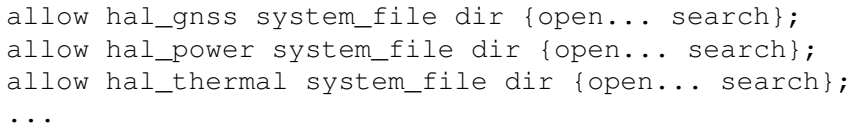

HAL, the "hardware abstraction layer," represents an important boundary between the core Android distribution and the efforts that OEMs make to port Android to their specific devices. These 23 HAL-related rules allow the same box, but it's unclear whether this was deliberate or accidental. Certainly, if a later analysis determines that system_server permissions need to be customized, or that this particular box needs to be denied, then each of these 23 rules would need to be changed. Many of these rules say nothing at all about system_server, at least not directly, but they impact it nonetheless.

How did this happen? This is a consequence of the overlapping rules in HAL-related macro functions and attributes, where macros sometimes even expand into other macros. The HAL subsystem would benefit from some degree of refactoring to simplify its security policies, redesigning it to avoid so much overlap.

Takeaway \#3: Most boxes only have a small number of rules, but a few boxes contain more than ten rules, which could be an obstacle for effective policy maintenance.

\subsection{Ratio of rule vs. box changes}

To further understand how commits affect the number of rules and the number of boxes, we plot the ratio $\Delta B / \Delta R$ for each commit, where $\Delta B$ is the number of added boxes, and $\Delta R$ is the number of added rules. If $\Delta B$ (or $\Delta R$ ) is negative, this means that a commit has reduced the number of boxes or rules, respectively. 
Figure 6 shows the results starting from July 2015we did not present earlier data due to the use of unconfineddomain. The average ratio is 120 , meaning that the "box" metric is more sensitive to changes. Moreover, we observed negative ratios, which means that the number of rules has increased (or decreased) while the number of boxes has decreased (or increased) - further evidence that their relation is non-linear.

For the peak indicated by A, the ratio is larger than 35,000 . We found that this is because Google added a new file type called vendor_file_type, and added file-related rules to the domain subject, which includes virtually all processes. Another peak, as indicated by B, has not affected the number of rules much; rather, a new permission map is added to existing rules. For instance, a rule

allow domain system_file: file \{execute read open\};

would become the following:

allow domain system_file: file \{execute read open map

Takeaway \#4: A change in the number of rules may not always lead to a corresponding change in the number of boxes.

\subsection{Summary}

To summarize, the box and rule metrics have an interesting and non-linear relationship; used in combination, anomalies and large changes directly point to interesting and relevant engineering changes in Android's history, such as the introduction and eventual elimination of Android's use of unconfineddomain.

By expanding rules to boxes, we gain an instrument that is very sensitive to policy changes. Even though the written rule changes might be small, the box changes can be enormous. This allows us to focus our attention on both local discontinuities, which point to specific significant patches made to the SEAndroid policy, as well as the broader multi-year trends in SEAndroid engineering. With our work, Google and OEMs could institute security policy metrics for SEAndroid. Our policy metrics could prove useful alongside other traditional software engineering metrics (e.g., lines of code, or bugs filed and fixed) to help Android project managers quantify the evolving complexity of their system. Any non-trivial changes in the metrics might imply significant policy differences, or perhaps even inadvertent policy misconfigurations.

\section{An Historical Analysis}

As we mentioned before, the metrics we've devised allow us to plot long-term patterns of the evolution of the SE-

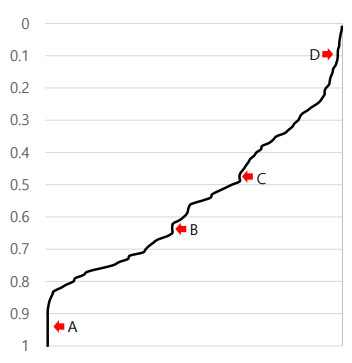

(a) Subjects

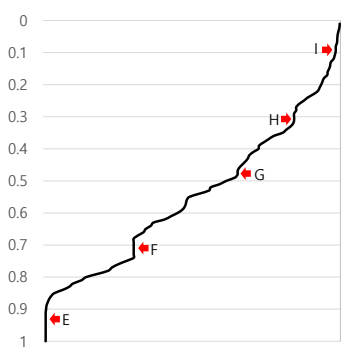

(b) Objects

\section{Figure 7. Cumulative distribution of the age of types.}

Android security policy. With these metrics, and the attendant timestamps, we can examine a number of other trends over time.

\subsection{The "age" of rules}

To start, we want a metric that speaks to the degree of turnover of the rules in SELinux policies. We can derive a given rule's "age" by identifying the Git commit when it was most recently changed. The longer a rule has been unchanged, the older it must be. Rather than using wallclock timestamps, we instead use the order of the commits. This means that, as the rate of commits has increased in recent years, our age metric will "speed up" to reflect the increased degree of activity.

Figures 7(a) and 7(b) show the cumulative distribution function diagrams of the age of each SEAndroid subject and object, respectively, across all commits on the master AOSP branch. The $x$-axis is the age of the type, and the $y$-axis is the cumulative distribution. This means that the types on the left side of the CDF are older, and types on the right side are more recent. The arrows "A" and " $\mathrm{E}$ " indicate the "oldest" subjects and objectives, respectively, which were created at the very beginning of the policy by NSA; they account for $20 \%$ of all the current types. The arrows " $\mathrm{C}$ " and " $G$ " indicate the introduction of the types for the hardware abstraction layer ("HAL") subsystem (e.g., hal_audio default and hal_drm_default). These types were introduced in a single commit by Google in October 2016.

At arrow "B", the types of system properties were separated into multiple types such as audio prop and bluetooth_prop. Before this commit, all the properties were associated with a single type, system_prop. This was presumably part of a privilege separation engineering push within Android. Perhaps as part of the same effort, at arrow "F", all the system services were separated into multiple types such as alarm_service and cpuinfo_service. Before this commit, all the system services were assigned to a 


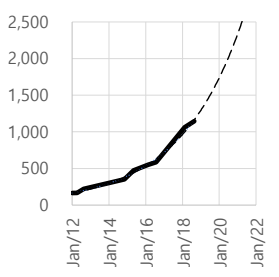

(a) Types

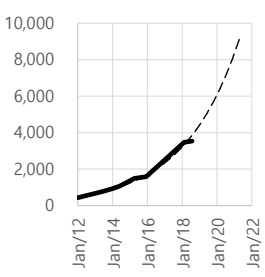

(b) Rules

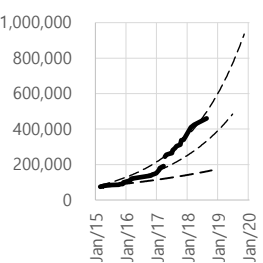

(c) Boxes
Figure 8. Fitted curves for types, rules, and boxes.

single type, system_service. In this commit, a new attribute temp_system_server_service was added and all the separated services were associated with the new attribute; they inherited all the rules for the existing system_service type. The temp_system_server_service attribute later disappeared, perhaps unsurprisingly, given it is a "temporary" name.

At arrow "H", a number of HAL related types were added in a single commit. In this case, the new types were related to a new HAL service which is now part of the version 8.0. Regions near arrows " $\mathrm{D}$ " and " $\mathrm{I}$ " indicate a radical increase of the types for Android, which are all part of the version 8.0 or later such as lowPAN (low-power wireless personal area network) for Android IoT devices and exported property feature for debugging annotations.

All of the commits at these labeled arrows after the initial NSA version were authored by Google. This suggests that other vendors are making only minor updates and tweaks, at least as far as their contributions to Android AOSP are concerned; large-scale engineering shifts are only happening at Google. We discuss more about different vendor contributions in Section 5.5 ,

Takeaway \#5: A non-trivial portion of the rules were present since the beginning, although the majority of the rules have gone through changes. Major events tend to affect a large number of rules, showing up as jump points in the curve.

\subsection{The increasing policy complexity}

As shown in Figures 3 and 7 , the complexity of the policy is exponentially increasing over time. Since the complexity is directly related to its maintainability, we would like to evaluate and estimate the growth pattern in more detail.

Figure 8 shows the linear scale fitted curves of three important indicators for policy complexity, which are the number of types, rules, and boxes. We generated this figure partly by extrapolating the future complexity increase based on the current change trends. For instance, extrapolating from the growth, the number of types and the number of rules might double from today (mid-2018) to mid-2020.

However, the fitted curve of the number of the boxes is different. As shown in Figure 8(c), we fit different segments of the graph with three different curves having different exponents. Not only is the number of boxes growing, the exponent of growth is growing! Super-exponential growth of our security policies cannot be a desirable attribute.

In Figure 8(c), we sampled important points after the "unconfined domain" period, which is separated to 3 different curves with different exponents and constants, because there are multiple jumps in the original curve due to the addition of new types, rules, and macros in every version release. For example, a big jump happened in 2017, due to the addition of HAL layer, as shown in Figure 7 The slopes of the fitted lines of all those three different curves are radically increasing.

This is a very important indication of the complexity increase- the exponent seems to be increasing, which suggests that the policy maintainability is getting more challenging as time goes on. Since there is virtually no maintenance manual or any public documentation about the policy, the device manufacturers, who need to customize the policy for their products, would face an enormous obstacle in terms of maintainability and evaluability for their product releases. Even if major contributors such as Google have consistently maintained internal documents, the trend still indicates that their maintainability is likely to get more difficult over time.

Takeaway \#6: The complexity of the SEAndroid policy is increasingly super-exponentially, which complicates maintainability and analysis.

\subsection{The effect of multiple branches}

Git is a distributed source-code management system, allowing separate organizations to maintain separate repositories with separate histories, merging those histories whenever they want. This has the curious property that the version of history that we see will change over time as alternative histories are merged or rebased into the mainline history. Among other issues, if two different branches are merged into one, we cannot distinguish which was the master and which was the merged branch without prior information; instead, we only can see that two branches merged. This means that tracking the "real" history of Android AOSP would require us to maintain snapshots of the entire Git repository's state, taken over time, rather than just examining the newest repository for its historical commits; this issue has been pointed out before by Bird et al. [5] as one of the potential downsides of Git mining and analysis. However, capturing all Git histories, continuously, is infeasible. To at least understand the impact of these issues, we analyzed our snapshot of the Android repository before and 


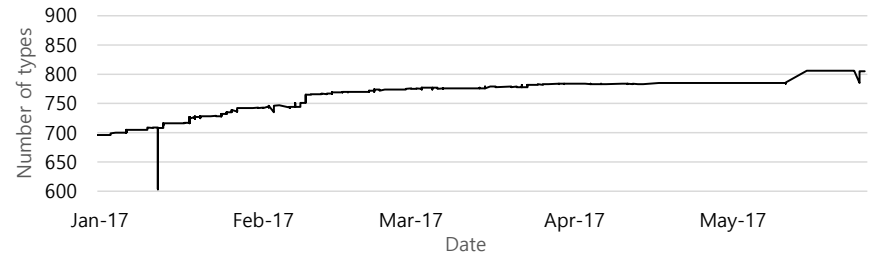

(a) Number of types captured in May 2017

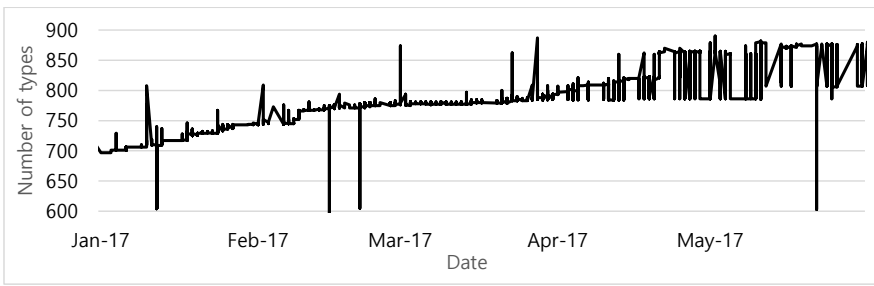

(b) Number of types captured in September 2017

\section{Figure 9. Comparison of the number of types in same period of time captured in different time}

after the "Oreo" Android releases in August 2017, where we observed more than 1,500 commits added in the span of a few days.

Figure 9 shows the two different curves with the number of SEAndroid types for the same period of time from January 2017 to May 2017, but reflecting the "pre-Oreo" and "post-Oreo" repository states. In Figure 9(a), we plot the "pre-Oreo" (late "Nougat") data from the end May 2017; in Figure 9(b), we plot the same time period from the AOSP repository as of September 2017. These two curves show the same upward trends but Figure 9(b)] shows significantly more noise. This is the result of a merge in the repository, performed at the end of July, with the merged commits originally committed in early 2017; these new commits only appeared on the master branch after the "Oreo" release, despite predating it. Since the graph uses the commit time as its $x$-axis, it shows fluctuations between the pre- and postOreo branch merger. Needless to say, branch merge events add additional complexity and noise to our data.

Most of the time-series graphs we derived in this paper have a similar square-shaped noise, likely due to merged branches. Because we cannot track private branches prior to their merger, it is not possible to distinguish which stream of the commit was the mainstream of the master branch before the merge. The timestamps are all we have, and thus we're stuck looking at interleaved time-series data, and thus the square-shaped noise. We can at least visually interpret the tops and bottoms of the square-shapes as representing the two original pre-merger commit streams.

This pattern shows that Google is continuing to de- velop Android internally, with mergers into the publicly visible AOSP only happening on major releases. While Android OEMs will hopefully have a more granular view of Google's efforts, any external developer trying to work with the SEAndroid security policy will observe these giant shifts and will have to debug and merge their changes with every Android release. Even for an Android OEM, Google's large changes presumably make it difficult for vendors to deviate from AOSP's SEAndroid policies in any meaningful way, as they would still have to rebase and/or merge their changes. For example, as we can see from the arrows in Figure 7, a single commit could introduce many new types, which would represent a significant integration challenge for an OEM trying to maintain a custom policy.

Takeaway \#7: The existence of multiple Git branches and merges introduce significant measurement challenges, as well as significantly impacting any third-party attempt to do their own work in SEAndroid.

\subsection{Case study: Stagefright}

We now turn to examine a newsworthy security event in Android's history and see if we can observe evidence in the evolution of the SEAndroid policy to respond to it.

To select the most suitable security events, we looked at 234 high-scoring Android CVEs in a third-party study [53]. 128 of them are related to hardware and driver issues, which are beyond the scope of SEAndroid. Next up are 62 vulnerabilities with the media framework. There are also 22 Adobe Flash vulnerabilities, but we cannot observe any artifacts of these in the SEAndroid policy, as Flash is not part of AOSP. Therefore, media framework vulnerabilities seem like a good place for a case study. The most widely publicized security event in the media framework, in particular, was the "Stagefright" vulnerability.

Stagefright was found and reported to Google by security researcher Joshua Drake in April 2015 [52]. Google shared the issue with device manufacturers in May 2015, and the vulnerability was publicly disclosed in August of that year. This vulnerability is a simple integer overflow bug in the libstageflight library which is used by the media framework. The attacker can inject carefully crafted malicious code, in a media file, via any application which uses Android's media API, allowing the attacker to have arbitrary malicious code execution in a context with elevated security privileges relative to any regular user-installed Android application. Google patched the integer overflow bug and distributed the patch in May 2015.

Figure 10 shows the number of the boxes and the number of the rules, in log scale, with media-related subjects and objects from January 2015 to September 2017. Red vertical bars indicate significant events in the history of the Stagefright vulnerability. The SEAndroid policy did not change 


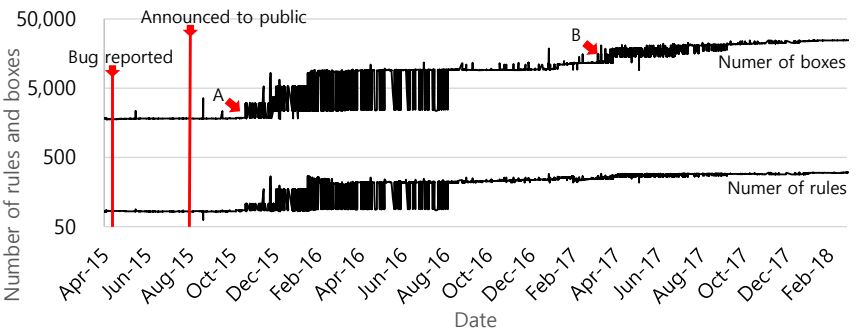

Figure 10. Number of boxes related to media.

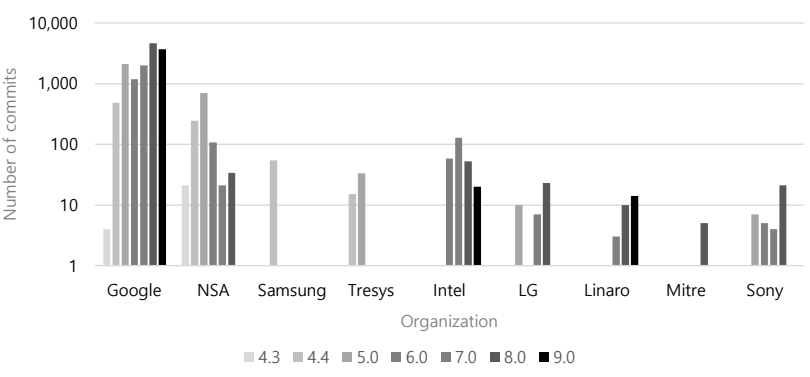

Figure 11. Number of commits contributed by each organization.

much during the initial period when the vulnerability was discovered, patched, and the fix was distributed. However, both the number of boxes and the number of the rules dramatically increased in early 2016 , shown at arrow "A". This was a result of separating the media server into multiple distinct services [10] such as mediaextractor, camera_server, mediadrm_server, and mediacodecservice, each with more limited privileges than the original monolithic service. Of note, all the media related services lost the permissions to write a normal file. Additionally, they lost the memexec permission.

Another jump, shown at arrow " $\mathrm{B}$ ", is related to the addition of rules related to a new audio_server service. While the number of rules changed was small, the number of boxes changed was much larger, indicating the importance and reach of the changes.

Takeaway \#8: SEAndroid policies are an essential mechanism for implementing privilege separation, refactoring monolithic services into smaller cooperating services with more limited permissions. These policy changes are more visible in our "box" metrics than when just looking at the number of rules.

\subsection{Contributor comparison}

Lastly, we perform a measurement to understand the composition of contributors to the SEAndroid policy. The Git history associates an email address with every commit. Ignoring the username and focusing on the domain name, we can then associate each commit with the author's organization. In Figure 11, we show the number of commits applied to each major Android release, as authored by each organization. As might be expected, the vast bulk of the commits are authored by Google, and Google's commit frequency is increasing over time, mirroring the exponentially growing numbers of rule and boxes.

The NSA is the second most frequent committer, with a spike of commits in the earlier Lollipop release, indicating some degree of NSA assistance as Google moved away from the "unconfined" domain design toward a more rigorous security policy.

We also see device and hardware manufacturers (Samsung, Intel, LG, Sony). Each of these vendors maintains its own private policies that they ship with their own devices. It is in a manufacturer's interest to contribute patches back to Google to avoid too much drift between Google's codebase and their own. Samsung stands out, in this regard, for having zero visible commits in the latest four versions of Android. (Perhaps Samsung contributed its policies to Google without sending Git pull requests. If so, a Google engineer would have merged their changes by hand, and the resulting commits would appear with a Google email address.)

The remaining organizations, Tresys, Linaro, and Mitre, represent two commercial consultancies and a Federally Funded Research and Development Center (FFRDC) that works extensively with the U.S. government. Tresys notably offers SELinux policy customization as a service for paying customers [46].

Takeaway \#9: Even though there are a number of nonGoogle contributors, most of their commits are simply fixing typos or adding missing simple rules which imply that only Google is leading the project. In addition, all the important policy changes such as all the arrow marks in Figures 3 and 7 are authored only by Google.

\section{Discussion}

Next, we discuss three items related to our measurement study: the Tizen system, the new Android Treble release, and Android for Work.

\subsection{SEAndroid vs. Smack}

As we have seen, the SEAndroid policy is getting more complex over time. Sophisticated policies may promise 
better security, but they also make it challenging to reason about the configuration, allowing innocent mistakes to creep into the design.

As a point of contrast, Tizen [28] uses a very different approach. Just like Android, Tizen is a Linux-based operating system targeted for mobile devices, and it uses a similar access control mechanism to SEAndroid called Smack [40]. The biggest difference is that Tizen doesn't overload Unix user IDs to separate applications from one another. Instead, all the apps for the same user use the same Unix user ID, but with Smack labels to isolate the applications.

We performed a small-scale experiment by counting the number of Smack rules in Tizen from its Git repository, and Figure 12 shows the result. We have removed the Smack rules related to specific applications to make a fair comparison with SEAndroid. We can see that the number of rules fluctuates across versions, but it stabilizes at roughly 2,000 in Tizen 3.0, which is radically smaller than the number of rules in SEAndroid.

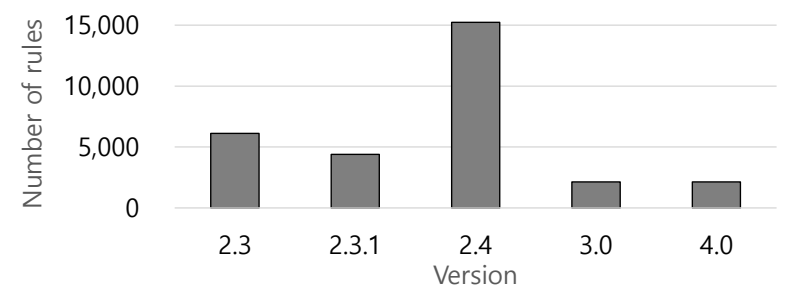

\section{Figure 12. Number of Smack rules in Tizen releases}

In Tizen 2.x, Smack is used for all the access control mechanisms including application isolation and API permissions, with the number of rules tightly related to the number of features. Consequently, the policy complexity is relatively high. The decrease in 2.3.1 and increase in 2.4 in Figure 12 is simply due to the removal and addition of many OS features [25]. Tizen 3.0 [26] featured a complete redesign of the Smack policy, providing only a minimal privilege separation for the system resources and each application [24]: Unix user IDs and Linux namespaces are used to isolate system services, and a new module called Cynara [23] was added to manage per-app API permissions (i.e., as in Android, users' grants of specific permissions to Tizen apps is no longer managed by the underlying Smack system but is instead managed in a separate system). The number of the rules in Tizen 3.0 and Tizen 4.0 is exactly the same: 2,134 rules, despite the otherwise significant changes between the two major releases, including support for IoT devices, and coding in C\#/.NET [27].

Although there is no "correct" approach to access control configuration, and we take no position on whether Ti- zen is fundamentally more or less secure than Android, Tizen demonstrates that MAC policies can be deployed without the staggering complexity of modern SEAndroid policies. Of course, the very lack of fine-grained permissions expressed in Tizen could as much represent the benefits of simplicity as the pitfalls of over-simplification. Regardless, the fact that both Android and Tizen are attempting to solve quite similar problems in radically different ways suggests that there may be lessons to draw from each to the benefit of the other.

\subsection{Android Treble}

In Android 8.0, Google introduced Treble [11] as a framework to separate platform and manufacturer features. Treble's main goal is to make it easier for Google to ship updated Android systems by creating a stable abstraction boundary between vendor features and the Android distributions from Google. If done properly, we should see a higher fraction of Android devices running recent releases of Android, improving security for Android users and simplifying the release engineering process for Android OEMs.

While a full summary of Treble is beyond the scope of this paper, Treble does have an impact on SEAndroid. In prior releases, Android vendors would start with Google's AOSP SEAndroid policy and make suitable modifications to support device-specific features, perhaps porting changes forward from release to release. Treble separates "vendor policies" from Google's own system policy. Both policies are separately compiled to a new common intermediate language (CIL), and are then combined into a single policy as part of the boot process. This allows Google to update its SEAndroid policy without vendor intervention.

The AOSP version of the SEAndroid policy is effectively the same as before, so it doesn't impact the continuing growth of SEAndroid policy complexity over time. But, now that vendor policies will be separated out, as more vendors ship devices with Android 8.0 or later, follow-on research to this paper will be able to look at vendor-specific firmware images as opposed to AOSP releases, and such future work will be able to make interesting comparisons between vendors. Some vendors will inevitably make huge changes while others change little or nothing. And, inevitably, some vendors will introduce security flaws by enabling too many permissions. Also, the new CIL format retains some macro and grouping structures, allowing it to be far more amenable to analysis than the compiled binary policies of earlier Android releases, where macros have been completely expanded. 


\subsection{Android for Work}

Android for Work is an enterprise security solution for "Bring-Your-Own-Device" (BYOD) environment, which was introduced in Android 5 and is still under active development. Android for Work supports separated runtime environments between work applications and personal applications. This requires extensive resource isolation, access control, and policy configuration. Knox, a similar BYOD solution introduced by Samsung [37], also used SELinux policies to support such access control. Android for Work would certainly be amenable for the same sort of analysis that we did in this paper, but the source code for it is not made available as part of AOSP. If it does become available in the future, its evolution over time would be an interesting subject to study, particularly as the Android for Work developers must necessarily respond to and integrate with changes in the larger SEAndroid policy.

\section{Related work}

Our work is most related to two lines of existing work: analyses of SEAndroid/SELinux policies, and general software-engineering analyses of Git repositories.

SEAndroid/SELinux policy analysis. Understanding software complexity is an important topic in the software engineering community, and a variety of useful metrics have been proposed over the years, such as cyclomatic complexity [31] and Halstead volume [32]. However, in the context of SEAndroid/SELinux policy, complexity measurements are much less studied; the dominant metric is simply the number of rules in the policy source code [36]. Researchers have also used formal verification on SELinux policies [14, 18, 38, 17, 2, 39], artificial intelligence, information flow integrity measurement [16, 15, 48], and functional tests [49]. Even machine learning techniques have been used to analyze policies based on SELinux denial logs from billions of devices [50].

Chen et al. [6] study the SEAndroid policy with the goal of identifying potential misconfigurations. They combine the SEAndroid mandatory policies with the discretionary policies embedded in the Android file system (i.e., Unix permission bits), giving them a more complete look at what is actually allowed or denied in practice. Both their work and ours present metrics and tools that might be useful in the Android development process. One important difference, however, is that we focus on quantifying the complexity of a policy snapshot, and on how the complexity evolves over time.

Git mining. Bird et al. [5] provide a comprehensive analysis on the pros and cons of Git mining [5]. Notably, one of the challenges they mention is the lack of a mainline repository when dealing with multiple Git branches.
Many other researchers have studied Git repositories (see, e.g., [44, 47, 13]). Negara et al. [33] use Git mining to detect patterns of code changes [33], German et al. [9] and Jiang et al. [20] analyze Linux kernel repositories' code over time. In our work, we apply similar concepts toward the study of SEAndroid's evolution.

\section{Conclusion}

In this paper, we have performed the first historical analysis of the SEAndroid policy to understand its evolution over time. We looked at both "rules" as written in the SEAndroid policy and the "boxes" those rules expanded to after processing all the macro and grouping operators. By plotting these metrics over time, we can observe the exponential growth in the complexity of SEAndroid policies, which will inevitably hit a brick wall of engineering complexity and require new and novel approaches to manage this complexity. Such approaches might lean on techniques from across many fields of computer science. For instance, we could utilize neverallow box as a policy "coverage" metric. Neverallow rules are used as assertion tools that policy engineer defines the boxes which should never be allowed, but as of November 2018, only 3\% of the possible boxes are covered by allow and neverallow boxes. Therefore, we could increase the coverage by measuring the current status and enhancing the number of the neverallow boxes.

\section{Acknowledgment}

We thank Stephen Smalley, Robert Williams and Tomasz Swierczek for valuable advice and answering many of our questions as well as the anonymous referees for their valuable feedback. This work was supported in part by NSF grants CNS-1801884, CNS-1409401, and CNS-1314492.

\section{References}

[1] Android Open Source Project. SEAndroid Policy Git Repository, 2018. https://android. googlesource.com/platform/system/ sepolicy

[2] M. Archer, E. Leonard, and M. Pradella. Analyzing security-enhanced Linux policy specifications. Technical report, Naval Research Lab Center For Computer High Assurance Systems, 2003.

[3] Armis, Inc. The Attack Vector "BlueBorne" Exposes Almost Every Connected Device, 2017. https:// wWw.armis.com/blueborne/ 
[4] L. Badger, D. F. Sterne, D. L. Sherman, K. M. Walker, and S. A. Haghighat. Practical domain and type enforcement for UNIX. In IEEE Symposium on Security and Privacy, pages 66-77, Oakland, CA, 1995. IEEE, IEEE.

[5] C. Bird, P. C. Rigby, E. T. Barr, D. J. Hamilton, D. M. German, and P. Devanbu. The promises and perils of mining Git. In 6th IEEE International Working Conference on Mining Software Repositories (MSR '09), pages 1-10, Vancouver, BC, Canada, 2009. IEEE, IEEE.

[6] H. Chen, N. Li, W. Enck, Y. Aafer, and X. Zhang. Analysis of seandroid policies: Combining mac and dac in android. In Proceedings of the 33rd Annual Computer Security Applications Conference, pages 553-565, Orlando,FL, 2017. ACM, ACM.

[7] N. Elenkov. Android Security Internals: An in-depth Guide to Android's Security Architecture. No Starch Press, 2014.

[8] A. P. Felt, E. Chin, S. Hanna, D. Song, and D. Wagner. Android permissions demystified. In 18th ACM Conference on Computer and Communications Security (CCS '11), pages 627-638, Chicago, IL, 2011. ACM.

[9] D. M. German, B. Adams, and A. E. Hassan. Continuously mining distributed version control systems: an empirical study of how Linux uses Git. Empirical Software Engineering, 21(1):260-299, 2016.

[10] Google. Media Framework Hardening, 2017. https://source.android.com/devices/ media/framework-hardening.

[11] Google. SELinux for Android 8.0, 2017. https: //source. android.com/security/ selinux/images/SELinux_Treble.pdf.

[12] Google. Android Open Source Project, 2018. https: / / source.android.com.

[13] G. Gousios, M.-A. Storey, and A. Bacchelli. Work practices and challenges in pull-based development: the contributor's perspective. In Software Engineering (ICSE), 2016 IEEE/ACM 38th International Conference on, pages 285-296, Austin, TX, 2016. IEEE, IEEE.

[14] B. Hicks, S. Rueda, L. St Clair, T. Jaeger, and P. McDaniel. A logical specification and analysis for SELinux MLS policy. ACM Transactions on Information and System Security (TISSEC), 13(3), 2010.
[15] T. Jaeger, R. Sailer, and U. Shankar. Prima: policyreduced integrity measurement architecture. In Proceedings of the eleventh ACM symposium on Access control models and technologies, pages 19-28, Lake Tahoe, CA, USA, 2006. ACM, ACM.

[16] T. Jaeger, R. Sailer, and X. Zhang. Analyzing integrity protection in the selinux example policy. In Proceedings of the 12th conference on USENIX Security Symposium-Volume 12, pages 5-5, Washington, DC, USA, 2003. USENIX Association, USENIX Association.

[17] T. Jaeger, R. Sailer, and X. Zhang. Resolving constraint conflicts. In Proceedings of the ninth ACM symposium on Access control models and technologies, pages 105-114, Yorktown Heights, NY, USA, 2004. ACM, ACM.

[18] T. Jaeger, X. Zhang, and A. Edwards. Policy management using access control spaces. ACM Transactions on Information and System Security (TISSEC), 6(3):327-364, 2003.

[19] X. Jiang and Y. Zhou. Dissecting android malware: Characterization and evolution. In 2012 IEEE Symposium on Security and Privacy, pages 95-109, San Francisco, CA, USA, 2012. IEEE, IEEE.

[20] Y. Jiang, B. Adams, and D. M. German. Will my patch make it? and how fast? case study on the Linux kernel. In 10th IEEE Working Conference on Mining Software Repositories (MSR), pages 101-110, an Francisco, CA, USA, 2013. IEEE, IEEE.

[21] M. La Polla, F. Martinelli, and D. Sgandurra. A survey on security for mobile devices. IEEE Communications Surveys \& Tutorials, 15(1):446-471, 2013.

[22] B. W. Lampson. Protection. ACM SIGOPS Operating Systems Review, 8(1):18-24, 1974.

[23] Linux Foundation. Security: Cynara, 2016. https: //wiki.tizen.org/Security: Cynara

[24] Linux Foundation. Smack three domain model, 2016. https://wiki.tizen.org/Security: SmackThreeDomainModel.

[25] Linux Foundation. Tizen 2.x security architecture, 2016. https://wiki.tizen.org/ Security/Tizen_2.X_Architecture.

[26] Linux Foundation. Tizen 3.0 security overview, 2016. https://wiki.tizen.org/Security/ Tizen_3.X_Overview 
[27] Linux Foundation. Tizen 4.0 Public M1 Release Notes, 2017. https://developer.tizen. org/tizen/release-notes/tizen-4.0public-m1.

[28] Linux Foundation. Tizen - An open source, standards-based software platform for multiple device categories, 2018. https://www.tizen.org/.

[29] P. Loscocco. Integrating flexible support for security policies into the Linux operating system. In Proceedings of the FREENIX Track: 2001 USENIX Annual Technical Conference, Boston, MA, 2001. USENIX association.

[30] L. Mahapatra. Android vs. ios: Whats the most popular mobile operating system in your country. International Business Times. Retrieved March, 1:2014, 2013.

[31] T. J. McCabe. A complexity measure. IEEE Transactions on Software Engineering, SE-2(4):308-320, Dec. 1976.

[32] J. C. Munson and T. M. Khoshgoftaar. The dimensionality of program complexity. In Proceedings of the 11th international conference on Software engineering, pages 245-253, Pittsburgh, PA, USA, 1989. ACM, ACM.

[33] S. Negara, M. Codoban, D. Dig, and R. E. Johnson. Mining fine-grained code changes to detect unknown change patterns. In Proceedings of the 36th International Conference on Software Engineering, pages 803-813, Hyderabad, India, 2014. ACM, ACM.

[34] Palo Alto Networks, Inc. Android Toast Overlay Attack: "Cloak and Dagger" with No Permissions, 2017. https://researchcenter. paloaltonetworks.com/2017/09/ unit42-android-toast-overlayattack-cloak-and-dagger-with-nopermissions/

[35] Redhat. SELinux/audit2allow, Fedora Wiki, 2015. http://fedoraproject.org/wiki/ SELinux/audit2allow.

[36] E. Reshetova, F. Bonazzi, T. Nyman, R. Borgaonkar, and N. Asokan. Characterizing SEAndroid policies in the wild. arXiv preprint arXiv:1510.05497, 2015.

[37] Samsung. Samsung Knox Security Solution, 2017. http://docs.samsungknox.com/ samsung-knox-whitepaper/SamsungKnox-Security-Solution-Whitepaper. pdf.
[38] B. Sarna-Starosta and S. D. Stoller. Policy analysis for Security-Enhanced Linux. In Proceedings of the 2004 Workshop on Issues in the Theory of Security (WITS), pages 1-12, Washington, DC, USA, 2004.

[39] A. Sasturkar, P. Yang, S. D. Stoller, and C. Ramakrishnan. Policy analysis for administrative role based access control. In Computer Security Foundations Workshop, 2006. 19th IEEE, pages 13-pp, Venice, Italy, 2006. IEEE, IEEE.

[40] C. Schaufler. The Simplified Mandatory Access Control Kernel, 2008. http://schauflerca.com/yahoo_site_admin/assets/ docs/SmackWhitePaper.257153003.pdf.

[41] Z. C. Schreuders, T. J. McGill, and C. Payne. Towards usable application-oriented access controls: Qualitative results from a usability study of SELinux, AppArmor and FBAC-LSM. International Journal of Information Security and Privacy, 6(1):57-76, 2012.

[42] S. Smalley. Configuring the SELinux policy. Technical Report 02-007, NAI Labs, 2005. https://www.nsa.gov/ resources/everyone/digital-mediacenter/publications/researchpapers/assets/files/configuringselinux-policy-report.pdf

[43] S. Smalley and R. Craig. Security enhanced (SE) Android: Bringing flexible MAC to Android. In NDSS, volume 310, pages 20-38, San Diego, CA, USA, 2013. Internet Society.

[44] M.-A. Storey, L. Singer, B. Cleary, F. Figueira Filho, and A. Zagalsky. The (r) evolution of social media in software engineering. In Proceedings of the on Future of Software Engineering, pages 100-116, Hyderabad, India, 2014. ACM, ACM.

[45] Tresys Technology. SELinux Policy Analysis Tools v4, 2017. https://github.com/ TresysTechnology/setools

[46] Tresys Technology. Tresys :: $\quad$ Security Enhanced Linux, 2017. http://www.tresys . com/ solutions/security-enhanced-linux

[47] B. Vasilescu, D. Posnett, B. Ray, M. G. van den Brand, A. Serebrenik, P. Devanbu, and V. Filkov. Gender and tenure diversity in github teams. In Proceedings of the 33rd Annual ACM Conference on Human Factors in Computing Systems, pages 3789-3798, Seoul, Republic of Korea, 2015. ACM, ACM. 
[48] H. Vijayakumar, G. Jakka, S. Rueda, J. Schiffman, and T. Jaeger. Integrity walls: Finding attack surfaces from mandatory access control policies. In Proceedings of the 7th ACM Symposium on Information, Computer and Communications Security, pages 75-76, Seoul, Republic of Korea, 2012. ACM, ACM.

[49] R. Wang, A. M. Azab, W. Enck, N. Li, P. Ning, X. Chen, W. Shen, and Y. Cheng. Spoke: Scalable knowledge collection and attack surface analysis of access control policy for security enhanced android. In Proceedings of the 2017 ACM on Asia Conference on Computer and Communications Security, pages 612624, Abu Dhabi, UAE, 2017. ACM, ACM.

[50] R. Wang, W. Enck, D. S. Reeves, X. Zhang, P. Ning, D. Xu, W. Zhou, and A. M. Azab. EASEAndroid: Automatic policy analysis and refinement for Security Enhanced Android via large-scale semi-supervised learning. In USENIX Security Symposium, pages 351366, Washington, DC, USA, 2015. USENIX Association.

[51] G. Zanin and L. V. Mancini. Towards a formal model for security policies specification and validation in the SELinux system. In Proceedings of the Ninth ACM Symposium on Access Control Models and Technologies, pages 136-145, Yorktown Heights, NY, USA, 2004. ACM, ACM.

[52] N. B. Zuk Avraham, Joshua Drake. Experts Found a Unicorn in the Heart of Android. Zimperium, July $2015 . \quad$ http: //blog.zimperium.com/experts-founda-unicorn-in-the-heart-of-android/.

[53] S. zkan. Google Android: List of Security Vulnerabilities. CVEdetails, 2018. https: //www.cvedetails.com/vulnerabilitylist/vendor_id-1224/product_id19997/Google-Android.html. 